\title{
Wer raucht, sollte auch (mäßig) trinken
}

— "Mäßiger Alkoholkonsum", so vermelden japanische Forscher auf dem US-Herzkongress, „könnte Raucher kurzfristig vor Herzproblemen schützen, indem es das HDL-Cholesterin erhöht." Doch was heißt hier mäßig? Die Diätleitlinien in den USA formulieren das so: Ein alkoholisches Getränk pro Tag für Frauen, und zwei für Männer Die japanischen Forscher aus Nagoya haben erstmals die Effekte von Rauchen und Trinken gemeinsam analysiert. Dazu hatten sie 25300 Männer ohne vorbestehende Herzkreislaufprobleme bezüglich ihrer Rauch- und Trinkgewohnheiten in Gruppen eingeteilt und dann 7,5 Jahre beobachtet, ob KHK, Infarkte oder Schlaganfälle auftraten.

Sie fanden heraus, dass das kardiovaskuläre Risiko von Rauchern, die kaum oder gar keinen Alkohol tranken (unter 20 g/d), mit zunehmender Zigarettenzahl pro Tag anstieg. Soweit ist das noch keine Überraschung. Aber: Wer mehr als $20 \mathrm{~g} / \mathrm{d}$ Alkohol trank (heftiger Alkoholismus ausgeschlossen), konnte rauchen, soviel er wollte, ohne dass das kardiovaskuläre Risiko anstieg.

Die Forscher bestimmten daraufhin das HDL-Cholesterin und fanden einen Durchschnittswert von $55 \mathrm{mg} / \mathrm{dl}$ bei Alkoholabstinenzlern und einen Wert von $61 \mathrm{mg} / \mathrm{dl}$ bei Menschen mit regelmäßigem Alkoholkonsum.

Fazit: Moderater Alkoholkonsum wirkt bei Rauchern protektiv. Wesentlich gesünder ist es aber, auf das Rauchen ganz zu verzichten: Raucher mit moderatem Alkoholkonsum sterben immer noch früher als Nichtraucher mit moderatem Alkoholkonsum.

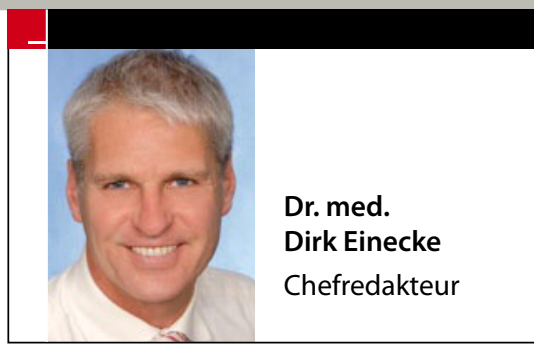

\section{KARDIOPROTEKTION (II)}

\section{Gartenarbeit schützt vor Herzattacken}

— Gute Nachricht im Frühling: Gartenarbeit verbessert die Herzfunktion und schützt langfristig vor Herzinfarkten.

Regelmäßiges Jäten, Sähen und Mähen verbessert die endotheliale Funktion in den Koronarien. Darüber hinaus senkt die Gartenarbeit im Laufe von über vier Jahren das Risiko für schwere kardiovaskuläre Komplikationen signifikant um 15\%.

Die Autoren hatten bei 111 Patienten, die wegen Angina oder anormaler nicht invasiver Herzbefunde einer Herzkatheteruntersuchung unterzogen wurden, nach Gabe von Papaverin, Acetylcholin und Nitroglyzerin mit Hilfe einer Doppler-Flussbestimmung die Endothelfunktion der Koronararterien untersucht. 82 Patienten gärtnerten regelmäßig, 29 taten dies nicht.

Obgleich sich Gartenfreunde und Gartenmuffel bezüglich anderer kardiovaskulärer Risikofaktoren nicht unterschieden, erwies sich das Koronarendothel der Gartenfreunde als signifikant geschmeidiger in Bezug auf die Blutflussaugmentation nach Vasodilatationsreiz. Ihre Chance, die nächsten vier Jahre ohne Herzattacken oder Schlaganfälle zu überleben, war signifikant größer. Gartenarbeit erwies sich als der einzige Prädiktor künftiger Koronarkomplikationen.

Die Autoren vermuten, dass das Gärtnern das autonome Nervensystem ausbalanciert, den Stress reduziert, und generell ein Ausdruck erhöhter Bereitschaft zu körperlicher Betätigung ist. nehmer ihr Routinesportprogramm absolvierten, "flimmerte" es im Schnitt 3,8mal; dagegen kam es während des Yogakurses, sprach, warnte der Autor davor, sämtliche Herzmedikamente abzusetzen; als Zusatzmaßnahme könnten die "Asanas" jedoch deutlich zu Buche schlagen. Zu konventionellen Maßnahmen bei Vorhofflimmern siehe unser Schwerpunkt ab S. 24. 